\title{
ARTIGO ORIGINAL Apreciação corporal e aspectos associados entre adolescentes e mulheres jovens
}

\author{
Body appreciation and associated factors among \\ adolescents and young women \\ Ana Carolina Soares Amaral' \\ https://orcid.org/0000-0003-2485-9111 \\ Aline de Souza Furtado Medeiros' \\ hitps:/lorid. orgy $0000-0001-8274-1168$ \\ Alcimara Campos de Morais Rodrigues Araújō \\ hitpsil/orididory/0000-002-7714-4825 \\ Alessandra Aparecida da Silva Ana' \\ hitps:/lorid. 0 org/000-0002-9737-2737 \\ Tassiana Aparecida Hudson² \\ hitps:/lorid. orgy/0000-0002-8562-3623 \\ Maria Elisa Caputo Ferreira² \\ https://orcid.org/0000-0002-3294-7560
}

\section{RESUMO}

Objetivo: Investigar os fatores que influenciam a apreciação corporal entre adolescentes e mulheres jovens. Métodos: As participantes $(n=242)$ responderam a questionários que avaliaram apreciação corporal, insatisfação corporal, influência sociocultural, atitudes alimentares e autoestima. Resultados: A análise de correlação revelou que todas as variáveis se associaram à apreciação corporal. O modelo de regressão linear múltipla apontou a satisfação corporal como principal preditor de maior apreciação corporal, seguido da autoestima, das atitudes alimentares e de menor influência da mídia. Conclusões: Esses resultados contribuem para o entendimento da imagem corporal positiva entre adolescentes e jovens brasileiras, apontando importantes questões e desafios para pesquisas futuras.

\section{ABSTRACT}

Objective: To investigate the factors that influence the body appreciation among adolescents and young women. Methods: Participants $(n=242)$ answered questionnaires that assessed body appreciation, body dissatisfaction, sociocultural influence, eating attitudes and self-esteem. Results: The correlation analysis revealed that all variables were associated with body appreciation. The multiple linear regression model pointed to body satisfaction as the

\section{Keywords}

Body image, positive psychology, sociocultural factors, self-esteem. main predictor of greater body appreciation, followed by self-esteem, eating attitudes and a lower influence of the media. Conclusions: These results contribute to the understanding of positive body image among Brazilian adolescents and youngsters, pointing out important issues and challenges for future research.

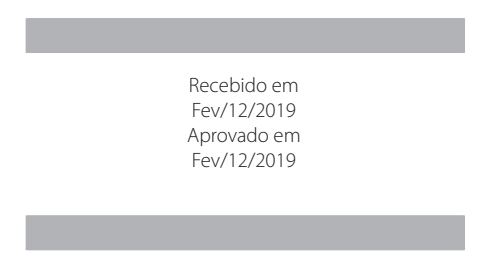

DOI: $10.1590 / 0047-2085000000220$

1 Instituto Federal de Educação, Ciência e Tecnologia do Sudeste de Minas Gerais - Campus Barbacena. 2 Universidade Federal de Juiz de Fora, Juiz de Fora, MG, Brasil.

Endereço para correspondência: Ana Amaral

Rua Monsenhor José Augusto, 204,

Bairro São José,

36205-018 - Barbacena, MG, Brasil

E-mail: ana.amaral@ifsudestemg.edu.br 


\section{INTRODUÇÃO}

A imagem corporal pode ser definida como a figura mental do corpo; em outras palavras, é o modo pelo qual o corpo se apresenta e como o sujeito o vivencia'. Definições atuais têm caracterizado imagem corporal não só como uma construção cognitiva, mas também como uma resposta dos desejos, emoções e interação social, caracterizando esse construto por duas grandes dimensões: a perceptiva, que define a precisão no julgamento do tamanho, forma e peso corporal; e a atitudinal, que envolve os componentes afetivo (emoções relacionadas ao corpo), cognitivo (pensamentos e crenças a respeito do próprio corpo) e comportamental (ações ou comportamentos relativos ao corpo) ${ }^{1}$.

A falta de adequação entre o corpo ideal e o percebido pode desencadear aumento da insatisfação corporal ${ }^{2}$. Dentre as principais influências sofridas pelos indivíduos com relação à imagem corporal, podem-se destacar as mídias, principalmente televisão e internet, que divulgam e valorizam o corpo perfeito. Sendo assim, a insatisfação corporal aumenta de acordo com a exposição de belos corpos pela mídia, o que atualmente tem provocado obsessão pelo corpo ideal ${ }^{3}$.

Por outro lado, pesquisadores contemporâneos têm buscado valorizar o construto da imagem corporal positiva, o que representa grande avanço nos estudos, visto que essa perspectiva considera a imagem corporal para além de seus aspectos patológicos 4 . Pode-se dizer que a imagem corporal positiva se caracteriza por sentimentos, pensamentos e atitudes que refletem a aceitação da sua aparência e elevado amor próprio, independentemente da sua forma física ${ }^{5}$, estando relacionada a numerosos índices de bem-estar, como satisfação com a vida e felicidade 4 .

A imagem corporal positiva pode ser entendida como amor abrangente e respeitoso pelo próprio corpo, permitindo a apreciação de sua beleza singular e suas funcionalidades. A demonstração de admiração com o corpo, incluindo as partes que são discordantes com as imagens idealizadas, a presença de sentimentos de felicidade e confiança com o corpo, destacando as qualidades em detrimento das imperfeições, a consciência relativa às necessidades corporais, a interpretação das informações recebidas, internalizando as positivas e recusando ou reformulando as negativas são características da imagem corporal positiva ${ }^{6}$.

Pode-se dizer que uma das facetas da imagem corporal positiva mais frequentemente avaliada tem sido a apreciação corporal7. Essa pode estar associada a diversos fatores, tais como autoestima ${ }^{8}$, satisfação com a vida, comer com atenção plena $a^{9,10}$, bem como inversamente correlacionada a comportamentos de checagem e evitação corporal e à internalização do ideal de aparência"1 . Além disso, pesquisadores têm descrito uma função protetiva da apreciação corporal. 0 chamado "filtro cognitivo de proteção" protege os indivíduos contra julgamentos negativos advindos de outras pessoas, bem como contra a influência da mídia, impedindo o desenvolvimento de alterações nos sentimentos relacionados ao próprio corpo e, consequentemente, prevenindo o desenvolvimento da insatisfação corporal4,12,13. Uma maior apreciação corporal também pode estar associada a uma percepção da aceitação do próprio corpo por amigos e familiares 5 .

Entre as escalas existentes para avaliação da apreciação corporal, a Body Appreciation Scale (BAS) é a mais comumente usada nas pesquisas sobre esse construto. Em seu desenvolvimento, Avalos et al. ${ }^{8}$ apontaram para a associação dos escores da BAS com sintomas de transtornos alimentares e índices de bem-estar como autoestima e otimismo. Para os autores, a imagem corporal positiva é um construto independente da imagem corporal negativa. Isso implica, por exemplo, que uma pessoa pode apresentar elevada apreciação corporal e, ao mesmo tempo, estar insatisfeita com algum aspecto em seu corpo ${ }^{11,14}$. Tiggemann e McCourt ${ }^{15}$ verificaram, em seu estudo com mulheres adultas, dois resultados principais que demonstram a independência desses construtos. Primeiramente, enquanto a apreciação corporal aumentou de acordo com o avanço da idade, a insatisfação corporal permaneceu estável. Em segundo lugar, a força da associação entre apreciação corporal e insatisfação corporal enfraqueceu com a idade, indicando que foi possível para as mulheres experimentarem algum nível de insatisfação corporal e, ao mesmo tempo, apreciarem e respeitarem seu corpo de outras formas. Tylka ${ }^{16}$ acrescenta que não se pode considerar que os achados sobre a imagem corporal negativa são aplicáveis e opostos à imagem corporal positiva, devendo, portanto, haver estudos específicos a fim de verificar tais associações.

Dessa forma, são necessárias investigações a respeito dos aspectos relacionados à imagem corporal positiva, a fim de fornecer embasamento para modelos teóricos robustos que expliquem o desenvolvimento desse construto, especialmente no contexto brasileiro, ainda carente desse tipo de investigação. Assim, o objetivo deste estudo foi verificar os fatores que mais fortemente influenciam a apreciação corporal em adolescentes e mulheres jovens.

\section{MÉTODOS}

\section{Participantes}

As alunas matriculadas nos cursos técnicos e superiores do Instituto Federal de Educação, Ciência e Tecnologia do Sudeste de Minas Gerais - Campus Barbacena foram convidadas a participar voluntariamente do estudo. As alunas que se recusaram a participar não foram incluídas no estudo e aquelas que deixaram de responder a algum questionário foram excluídas.

A amostra total do presente estudo foi composta por 242 adolescentes e mulheres jovens, com idade variando entre 
14 e 30 anos $(M=18,97 ; D P=2,90)$. Entre as participantes, $47,1 \%(n=114)$ apresentavam idade entre 14 e 18 anos e $52,9 \%(n=128)$ possuíam entre 19 e 30 anos.

\section{Instrumentos}

Os valores do peso e estatura foram informados pelas participantes, para que fosse possível calcular o índice de massa corporal (IMC; kg/m²). A classificação do estado nutricional das participantes seguiu as orientações da Organização Mundial de Saúde ${ }^{17}$. Para as adolescentes, foram adotados os percentis propostos para meninas de acordo com a idade, sendo consideradas com baixo peso aquelas abaixo do percentil 5, como eutróficas aquelas com IMC entre os percentis 5 e 85, e as meninas com IMC superior ao percentil 85 foram consideradas em sobrepeso. Para as adultas, considerou-se como baixo peso aquelas com IMC inferior a $18,5 \mathrm{~kg} / \mathrm{m}^{2}$, eutróficas aquelas com IMC entre 18,5 e $25 \mathrm{~kg} / \mathrm{m}^{2}$, e com sobrepeso as que apresentaram IMC entre 25 e $30 \mathrm{~kg} / \mathrm{m}^{2}$.

Para avaliação da apreciação corporal, utilizou-se a BAS. Essa escala, criada por Avalos et al. ${ }^{8}$, avalia a apreciação corporal por meio de 13 itens, em escala likert de pontos, variando de 1 (nunca) a 5 (sempre). A pontuação varia de 13 a 65 pontos e quanto maior o escore final, maior é a apreciação corporal do indivíduo. Para o presente estudo, utilizou-se a versão do instrumento validada para adultos brasileiros por Swami et al. ${ }^{18}$. As propriedades psicométricas desse instrumento também foram confirmadas para adolescentes, por Moreira et al. ${ }^{19}$. A consistência interna da escala entre as participantes da presente pesquisa foi de 0,91.

A insatisfação corporal foi avaliada por meio do Body Shape Questionnaire (BSQ), criado por Cooper et al. ${ }^{20}$. Esse questionário é composto por 34 itens em escala likert de pontos (1: nunca; 6: sempre) e seu escore varia de 34 a 204 pontos. Para o BSQ, quanto maior o escore obtido, maior a insatisfação corporal. Para o presente estudo, utilizaram-se duas versões desse instrumento, aplicadas de acordo com a faixa etária. São elas: a versão validada para adolescentes de 10 a 18 anos por Conti et al. ${ }^{21}$ e a versão validada para universitários brasileiros por Di Pietro e Silveira ${ }^{22}$. O BSQ obteve consistência interna de 0,96 para a amostra deste estudo.

O Questionário de Atitudes Socioculturais em Relação à Aparência-3 (SATAQ-3) desenvolvido por Thompson et al. ${ }^{23}$ foi utilizado para avaliar a influência sociocultural em relação à imagem corporal. O instrumento possui 30 itens, em escala likert de pontos, variando de 1 (discordo totalmente) a 5 (concordo totalmente). A soma dos pontos varia de 30 a 150, e a pontuação representa proporcionalmente a influência sociocultural sobre a imagem corporal. O SATAQ-3 foi validado para adolescentes ${ }^{24}$ e jovens brasileiros ${ }^{25}$. Para a amostra deste estudo, a consistência interna avaliada pelo alfa de Cronbach foi de 0,93.

A avaliação da influência dos pais e dos amigos foi realizada pelas subescalas correspondentes da Escala de Influên- cia dos Três Fatores (EITF). Essa escala foi desenvolvida originalmente por Keery et al. ${ }^{26}$ e a avaliação de suas qualidades psicométricas para a população jovem brasileira foi realizada por Amaral et al. ${ }^{27}$. As subescalas utilizadas nesse estudo totalizam 29 itens, em escala likert de pontos (1: sempre; 5: nunca). O escore total é calculado pela soma das respostas e, para esse estudo, variou de 29 a 145 pontos. Para a EITF, quanto menor o escore, maior a influência dos fatores sobre a imagem corporal. Apesar de ter sido validada apenas para adultos jovens, essa escala já foi utilizada previamente em uma amostra numerosa de adolescentes ${ }^{28}$, a partir da indicação de bom nível de consistência interna também nessa população. Seguindo essa direção, a consistência interna para a amostra desta pesquisa foi de 0,91.

O Eating Attitudes Test-26 (EAT-26) foi utilizado para a avaliação das atitudes alimentares das participantes. Essa escala foi desenvolvida por Garner et al. ${ }^{29}$ e validada para meninas brasileiras por Bighetti et al. ${ }^{30}$. O EAT-26 é composto por 26 questões, em escala likert de pontos (3: sempre; 2: muitas vezes; 1: frequentemente; 0 : poucas vezes, quase nunca e nunca). O escore total varia entre 0 e 78 pontos e, quanto maior o valor obtido, maior a frequência de atitudes alimentares inadequadas ("comer transtornado"). Escores superiores a 21 são indicativos de atitudes alimentares transtornadas. Entre as participantes do presente estudo, obteve-se consistência interna de 0,83.

Por fim, a Escala de Autoestima de Rosenberg ${ }^{31}$ (EAR) foi utilizada para avaliação da autoestima global das participantes. A EAR é composta por 10 itens, em escala likert variando de 1 (discordo totalmente) a 4 (concordo totalmente), e quanto maior o escore total, maior a autoestima do entrevistado. Essa escala foi validada para a população brasileira de 10 a 30 anos por Hutz e Zanon ${ }^{32}$ e a consistência interna do instrumento para as participantes do presente estudo foi de 0,92 .

\section{Procedimentos}

Após a autorização da instituição para a realização da pesquisa, foram convidadas para participarem do estudo as adolescentes e jovens adultas matriculadas nos cursos técnicos e superiores do IF Sudeste MG - Campus Barbacena, e foram entregues os Termos de Consentimento Livre e Esclarecido - TCLE (que deveriam ser assinados por um responsável, para as adolescentes menores de 18 anos). Foram incluídas na presente pesquisa apenas as adolescentes e jovens adultas que entregaram o TCLE assinado. As menores deveriam, ainda, assinar o Termo de Assentimento Livre e Esclarecido (TALE), concordando em participar voluntariamente do estudo.

A aplicação dos questionários foi conduzida em um único momento, em salas de aula, e todas as voluntárias responderam de forma individual aos questionários e a um cabeçaIho de identificação e de dados antropométricos. 
Os resultados desse estudo fazem parte da pesquisa "Imagem corporal dos adolescentes: diagnóstico e intervenção", cujo projeto foi aprovado pelo Comitê de Ética e Pesquisa em Seres Humanos da Universidade Federal de Juiz de Fora (UFJF), sob Parecer no 228.386. Sua execução está de acordo com as normas da Resolução no 466/12, do Conselho Nacional de Saúde.

\section{Análise dos dados}

Inicialmente, foi realizada a análise descritiva das variáveis e verificada a suposição de normalidade. Constatada a violação de normalidade (Kolmogorov-Smirnov $<0,05$ ), utilizou-se o teste $U$ de Mann-Whitney para verificação de diferenças nas variáveis entre os grupos etários e a correlação de Spearman para avaliação da associação entre as variáveis do estudo. O teste qui-quadrado foi utilizado para verificar a existência de diferenças entre os grupos etários para as classificações do estado nutricional. A análise de regressão linear múltipla forward foi utilizada a fim de investigar os preditores da apreciação corporal. O pressuposto de colinearidade foi avaliado por meio do VIF (variance inflation factor), sendo valores desejáveis os menores que 5.

As análises foram feitas utilizando-se o software SPSS v. 21.0, adotando nível de significância de 95\% ( $p<0,05)$.

\section{RESULTADOS}

Em um primeiro momento, verificou-se que não houve diferenças entre os grupos etários para nenhuma das variáveis avaliadas $(p>0,05)$. Dessa forma, todas as análises subsequentes foram realizadas com toda a amostra ( $n=242)$. Quanto à classificação do estado nutricional, identificaram-se 4,1\% de participantes com baixo peso, 82,2\% eutróficas e 13,3\% com sobrepeso, não havendo diferenças na distribuição do estado nutricional entre as faixas etárias para o teste qui-quadrado $(p>0,05)$.

A análise de correlação entre a idade e as demais variáveis revelou associação significante apenas para a apreciação corporal ( $r=0,154$; $p=0,009)$ e a autoestima ( $r=0,213$; $p<0,001$ ), indicando que quanto mais velhas, maiores a apreciação corporal e a autoestima das participantes.

A análise descritiva das variáveis do estudo, bem como os coeficientes de correlação com a apreciação corporal, estão apresentados na tabela 1.

A análise de correlação revelou que todas as variáveis se correlacionaram significantemente com a apreciação corporal $(r>0,13 ; p<0,05)$. A tabela 2 mostra os resultados obtidos para a regressão linear múltipla. Todas as variáveis inseridas obtiveram VIF inferior a 5, sendo incluídas na regressão. No modelo obtido, a influência de pais e amigos, avaliada pela EITF, e o IMC não apresentaram relação significante com a apreciação corporal.
Tabela 1. Análise descritiva das variáveis do estudo e correlação de Spearman com a apreciação corporal

\begin{tabular}{lcc}
\hline Variáveis & $M(D P)$ & $R$ \\
\hline Apreciação corporal (BAS) & $44,90(10,43)$ & - \\
IMC & $21,73(3,58)$ & $-0,128^{*}$ \\
Insatisfação corporal (BSQ) & $81,79(34,37)$ & $-0,630^{* *}$ \\
Influência da mídia (SATAQ-3) & $79,68(24,44)$ & $-0,464^{* *}$ \\
Influência de pais e amigos (EITF) & $113,82(17,54)$ & $0,268^{* *}$ \\
Autoestima (EAR) & $29,73(6,20)$ & $0,684^{* *}$ \\
Atitudes alimentares (EAT-26) & $13,71(9,81)$ & $-0,392^{* *}$ \\
\hline${ }^{*}<0,05 * * * p<0,01$. & &
\end{tabular}

Tabela 2. Resultados da Regressão Linear Múltipla utilizando a apreciação corporal como variável dependente

\begin{tabular}{lcc}
\hline Variáveis & Beta padronizado & $p$ \\
\hline Insatisfação corporal & $-0,467$ & 0,000 \\
Autoestima & 0,458 & 0,000 \\
Atitudes Alimentares & 0,180 & 0,009 \\
Influência da mídia & $-0,132$ & 0,027 \\
$R$ & & 0,774 \\
$R^{2}$ & & 0,599 \\
$F(p)$ & \multicolumn{2}{c}{$74,629(0,000)$} \\
\hline
\end{tabular}

\section{DISCUSSÃO}

Esse estudo buscou avaliar a influência de alguns fatores sobre a apreciação corporal, dimensão importante da imagem corporal positiva. De acordo com os resultados, as variáveis independentes selecionadas estiveram significantemente correlacionadas com a apreciação corporal, e o modelo de regressão linear apontou que elas foram capazes de explicar $60 \%$ da variância do desfecho.

A partir dos resultados encontrados, não foram observadas diferenças nas variáveis, de acordo com a faixa etária. Entretanto, ao analisar as variáveis de forma linear (análise de correlação), notou-se associação fraca, porém significante, entre a idade e a apreciação corporal, indicando que quanto mais velhas, maior a apreciação corporal das participantes. Ao relacionar a apreciação corporal com a idade, Tiggemann e McCourt ${ }^{15}$ identificaram que mulheres mais velhas tendem a ter maior apreciação corporal, resultado semelhante ao notado no presente estudo. Deve-se considerar, ainda, que no estudo de Tiggemann e McCourt ${ }^{15}$ o intervalo de idade foi mais abrangente (18 a 75 anos), tendo sido identificada a maior apreciação nas mulheres de 51 a 75 anos, em comparação com as adolescentes e jovens adultas.

Destaca-se que o fato de a idade ter se associado significantemente à apreciação corporal mas não à insatisfação reforça a teoria de que esses construtos são independentes, e não apenas opostos. Apesar de as participantes terem apre- 
sentado maior apreciação corporal quanto maior a idade, não foi notada redução na insatisfação corporal. A despeito desse resultado, notou-se associação significante entre essas duas variáveis ( $r=-0,630)$, com associação inversa; ou seja, indivíduos com maior apreciação corporal apresentaram, também, menor insatisfação com o próprio corpo. Importante destacar que, apesar de serem construtos diferentes e independentes, outros estudos têm apontado para a associação entre essas variáveis. Andrew et al.12, utilizando uma escala visual analógica na avaliação da insatisfação corporal, identificaram que as participantes com baixa e média apreciação corporal (avaliada pela BAS) apresentaram, também, maior insatisfação corporal. Correlação semelhante foi notada no estudo de validação da versão espanhola da BAS, o qual verificou associação moderada e significante da apreciação corporal e da insatisfação corporal, apesar de ter utilizado um instrumento diferente do $\mathrm{BSQ}^{33}$. De forma semelhante, Moreira et al. ${ }^{19}$ no estudo de validação da escala para adolescentes brasileiros também verificaram essa associação, com adolescentes satisfeitos apresentando, também, maior apreciação corporal.

Essa associação foi corroborada pelos resultados da regressão, que apontaram menor insatisfação corporal como principal preditor de um aumento na apreciação corporal. De forma semelhante, Tiggemann e McCourt ${ }^{15}$ apontaram a insatisfação corporal como forte preditor da apreciação corporal entre mulheres adultas, independentemente da idade. Entretanto, o fato de que a insatisfação corporal foi notada como forte preditor da apreciação corporal não pode ser compreendido como que esses construtos sejam similares. Wood-Barcalow et al. ${ }^{34}$, entrevistando jovens universitárias americanas, identificaram a interdependência desses construtos nas falas das participantes, e não que ambos são mutuamente exclusivos. Segundo os autores, apesar de algumas participantes não estarem totalmente satisfeitas com sua aparência, elas não deixavam esse sentimento sobrepujar sua apreciação corporal geral.

A autoestima, por sua vez, foi a variável com índice de correlação mais alto com a apreciação corporal $(r=0,684)$, indicando que quanto maior a autoestima, maior também foi a apreciação corporal. Ao avaliarem-se os resultados da regressão, uma maior autoestima apresentou-se como preditor significante do aumento da apreciação corporal. Esse resultado corrobora os achados de Skorek et al. ${ }^{35}$, os quais identificaram a autoestima como mediador completo da relação entre traços de personalidade e a estima corporal. Além disso, essa relação já é bem documentada na literatura, sendo a medida da autoestima utilizada, inclusive, como medida de validade das escalas de avaliação da apreciação corporal ${ }^{8,36}$. Essa relação pode ser explicada, ainda, pelo fato de a autoestima envolver, entre outros aspectos, o autoconceito positivo, que se configura como um facilitador em potencial para o desenvolvimento da imagem corporal positi- va, atuando como fator protetor contra eventos que possam prejudicar a autoimagem ${ }^{37}$

As atitudes alimentares, avaliadas pelo EAT, apresentaram correlação negativa e significante com a apreciação corporal $(r=-0,392)$. Relação semelhante foi apontada no estudo de validação da BAS, no qual os autores verificaram associação negativa entre esses construtos ${ }^{8}$. Entretanto, essa variável se comportou de forma inesperada no modelo de regressão, sendo preditor significante da apreciação corporal, mas no sentido positivo. Ou seja, no modelo avaliado, a adoção de comportamentos alimentares inadequados foi proporcional à apreciação corporal. De forma exploratória, verificou-se que participantes com pontuação inferior ao ponto de corte do EAT-26 ( $n=182)$ apresentaram maiores escores na BAS que aquelas com pontuação superior a 21 ( $n=51$; $p<0,05)$. Entretanto, ao se avaliar a amplitude da pontuação, os escores obtidos para a BAS pelas participantes variaram de forma semelhante, de 21 a 64 pontos para as primeiras $(M=46,85)$ e 21 a 62 para as seguintes $(M=37,92)$. Esse fato demonstra que é possível que a relação entre essas variáveis - atitudes alimentares e apreciação corporal - não seja linear. Ao analisar o conceito da imagem corporal positiva, uma das características desse construto é o estabelecimento de uma relação de cuidado com o corpo, tendo, por exemplo, uma alimentação saudável, que auxilie no bom funcionamento do corpo ${ }^{16}$. Assim, atitudes como determinar quando e o quanto comer e comer alimentos que são mais atrativos e saudáveis são exemplos de autocuidado corporal e que, por sua vez, estão presentes em algumas questões do instrumento utilizado no presente estudo para a identificação de atitudes alimentares transtornadas (por exemplo, "Evito, particularmente, os alimentos ricos em carboidratos"; "Evito comer alimentos que contenham açúcar", entre outros). Dessa forma, a relação proporcional apontada pelo modelo de regressão pode indicar que as atitudes alimentares de restrição calórica ou preocupação com os alimentos, por exemplo, representam uma apreciação corporal elevada por esse comportamento refletir um cuidado com o corpo, e não uma atitude deletéria à saúde.

De forma semelhante, a influência da mídia apresentou-se significantemente correlacionada à apreciação corporal $(r=-0,464)$. Ainda, de acordo com o modelo de regressão linear múltipla, uma menor influência da mídia foi preditora significante de maior apreciação corporal. De acordo com Tylka ${ }^{16}$, a imagem corporal positiva está relacionada à capacidade de estar consciente da natureza irreal das imagens divulgadas na mídia, bem como rejeitar imagens e mensagens baseadas em um padrão corporal. Nesse sentido, Andrew et al. ${ }^{12}$ verificaram que as jovens com maior apreciação corporal foram menos afetadas pela exposição a imagens midiáticas, apontando para o fator protetivo da imagem corporal positiva contra a influência sociocultural, conforme já havia sido apontado por Halliwell ${ }^{4}$. Ademais, pessoas com 
uma imagem corporal mais positiva evitam ou minimizam a exposição ao ideal corporal apresentado pela mídia ${ }^{16}$, o que justifica os resultados encontrados no presente estudo.

Em contrapartida, a influência dos pais e dos amigos esteve significantemente correlacionada à apreciação corporal, indicando que as participantes com maiores níveis de apreciação corporal apresentavam, também, menor influência dessas fontes ( $r=0,277)$. Essa associação não foi confirmada no modelo de regressão linear, e a EITF não apresentou relação significante com a apreciação corporal. Esses resultados vão de encontro aos achados de Andrew et al. ${ }^{5}$, os quais identificaram que a aceitação percebida do corpo por pais e amigos induziu a uma maior aceitação do próprio corpo entre adolescentes. Uma possível explicação para esse resultado está no conceito da imagem corporal positiva. Entre suas características, está o fato de não condicionar a própria aceitação às opiniões dos pares e pessoas próximas. O chamado "filtro cognitivo de proteção", característico de pessoas com elevada apreciação corporal, implica a não alteração dos sentimentos relacionados ao próprio corpo, mesmo após julgamentos negativos advindos de outras pessoas ${ }^{13}$.

Finalmente, verificou-se que o IMC esteve negativamente correlacionado à apreciação corporal, indicando que quanto maiores os valores de IMC, menor a apreciação corporal das participantes. Resultados semelhantes foram apontados por Swami et al. ${ }^{38}$, ao correlacionarem algumas variáveis com a apreciação corporal, com o IMC apresentando associação negativa com essa variável. Entretanto, o modelo de regressão linear obtido não apontou o IMC como preditor significante da apreciação corporal. Ademais, para Tylka ${ }^{16}$, uma das características da imagem corporal positiva é o conceito amplo de beleza, o que implica perceber uma diversidade de pesos, formas e aparências como belos, o que justifica o fato de a apreciação corporal não estar relacionada diretamente ao IMC do sujeito.

Ao interpretar os resultados do presente estudo, é preciso considerar algumas limitações. Em primeiro lugar, devido ao delineamento transversal, não é possível estabelecer relações de precedência temporal entre os fatores. Destaca-se, entretanto, que as associações verificadas fornecem informações importantes sobre os aspectos relacionados à apreciação corporal, dada a carência de investigações sobre esse construto. Em segundo lugar, é necessária cautela ao se ampliar os resultados encontrados para outras populações, pois a amostra procedeu de uma única cidade, do interior de Minas Gerais. Apesar disso, considera-se que a amostra obtida foi numerosa, apontando para características importantes dos construtos avaliados. Vale destacar, ainda, que, apesar do esforço em utilizar apenas escalas com adequadas qualidades psicométricas para a totalidade do público-alvo da pesquisa, a escala selecionada para avaliação da influência dos pais e dos amigos (EITF) foi validada apenas para jovens brasileiras. Argumenta-se, entretanto, que essa é a úni- ca escala disponível no Brasil para avaliação desse construto e apresentou consistência interna adequada para a amostra do presente estudo. A despeito das limitações apresentadas, o presente estudo cumpre seu papel, ao contribuir para a compreensão da associação de aspectos importantes com a apreciação corporal em adolescentes e jovens brasileiras, tendo em vista a lacuna nos estudos da literatura nacional que investiguem as dimensões da imagem corporal positiva e sua relação com diferentes fatores.

\section{CONCLUSÕES}

Os resultados apontaram menor insatisfação corporal como a variável de maior poder explicativo sobre a apreciação corporal, seguida da autoestima, das atitudes alimentares e menor influência da mídia. Considerando a carência de estudos brasileiros que avaliem a apreciação corporal e sua associação a outras variáveis, encorajam-se estudos com amostras diversificadas, incluindo participantes do sexo masculino, mais numerosas e em diferentes contextos sociais, a fim de aprofundar as relações encontradas no presente estudo, bem como aquelas cujas hipóteses não foram confirmadas, como é o caso da influência dos pais e amigos e das atitudes alimentares.

\section{CONTRIBUIÇÕES INDIVIDUAIS}

Ana Carolina Soares Amaral - Contribuiu em todas as etapas de realização do estudo, incluindo a concepção e desenho do estudo, coleta e análise dos dados, interpretação dos resultados, revisão de literatura, elaboração do manuscrito e revisão e aprovação da versão final a ser publicada.

Aline de Souza Furtado Medeiros, Alcimara Campos de Morais Rodrigues Araújo e Alessandra Aparecida da Silva Ana - Contribuíram na coleta e análise dos dados, interpretação dos resultados, revisão de literatura, elaboração do manuscrito e aprovação da versão final a ser publicada.

Tassiana Aparecida Hudson - Contribuiu na concepção e desenho do estudo, coleta e análise dos dados, interpretação dos resultados, elaboração do manuscrito e revisão e aprovação da versão final a ser publicada.

Maria Elisa Caputo Ferreira - Contribuiu na concepção e desenho do estudo, interpretação dos resultados e revisão e aprovação da versão final a ser publicada.

\section{CONFLITOS DE INTERESSE}

Os autores não possuem conflitos de interesse a serem declarados. 


\section{AGRADECIMENTOS}

Às participantes do estudo. Ao Instituto Federal de Educação, Ciência e Tecnologia do Sudeste de Minas Gerais e ao Conselho Nacional de Desenvolvimento Científico e Tecnológico (CNPq), pela concessão de bolsas de iniciação científica e de apoio ao pesquisador concedidas a esta pesquisa.

\section{REFERÊNCIAS}

1. Ferreira MEC, Castro MR, Morgado FFR. Imagem corporal: reflexões, diretrizes e práticas de pesquisa. Juiz de Fora: Editora da UFJF; 2014.

2. Claro RM, Santos MAS, Campos M0. Imagem corporal e atitudes extremas em relação ao peso em escolares brasileiros (PeNSE 2012). Rev Bras Epidemiol. 2014;17:146-57.

3. Frois E, Moreira J, Stengel M. Mídias e a imagem corporal na adolescência: 0 corpo em discussão. Psicol Estud. 2011;16(1):71-7.

4. Halliwell E. The impact of thin idealized media images on body satisfaction: does body appreciation protect women from negative effects? Body Image. 2013;10(4):509-14.

5. Andrew R, Tiggemann M, Clark L. Predictors and Health-Related Outcomes of Positive Body Image in Adolescent Girls: A Prospective Study. Dev Psychol. 2016;52(3):463-74.

6. Tylka TL, Wood-Barcalow NL. What is and what is not positive body image? Conceptual foundations and construct definition. Body Image. 2015;14:118-29.

7. Webb JB, Wood-Barcalow NB, Tylka TL. Assessing positive body image: Contemporary approaches and future directions. Body Image. 2015;14:130-45.

8. Avalos L, Tylka TL, Wood-Barcalow N. The Body Appreciation Scale: Development and psychometric evaluation. Body Image. 2005;2(3):285-97.

9. Augustus-Horvath CL, Tylka TL. The acceptance model of intuitive eating: A comparison of women in emerging adulthood, early adulthood and middle adulthood. J. Couns. Psychol. 2011:58:110-25

10. Swami V, Tran US, Stieger S, Voracek M, The YouBeauty.com Team. Associations between women's body image and happiness: Results of theYouBeauty.com Body Image Survey (YBIS). J. Happiness Stud. 2015;16(3):705-18.

11. Halliwell E. Future directions for positive body image research. Body Image. 2015;14:177-89

12. Andrew R, Tiggemann M, Clark L. The protective role of body appreciation against mediainduced body dissatisfaction. Body Image. 2015;15:98-104

13. Tylka TL, Kroon van Diest AM. Protective Factors. In: Levine MP, Smolak L (Eds.). The Wiley Handbook of Eating Disorders. Nova Jersey: Wiley-Blackwell; 2015. p. 4 30-444.

14. Pope M, Corona R, Belgrave FZ. Nobody's perfect: A qualitative examination of African American maternal caregivers' and their adolescent girls' perceptions of body image. Body Image. 2014;11:307-17.

15. Tiggemann M, McCourt A. Body appreciation in adult women: Relationships with age and body satisfaction. Body Image. 2013;10(4):624-7.

16. Tylka TL. Positive psychology perspectives on body image. In: Cash TF, Smolak L (Eds.) Body image: A handbook of science, practice, and prevention. New York: Guilford Press; 2011. p. 56-64.

17. World Health Organization (WHO). Phisical Status: the use and interpretation of anthropometry. WHO Technical Report Series n. 854. Geneva: WHO; 1995.

18. Swami V, Campana ANNB, Ferreira L, Barrett S, Harris AS, Tavares MCGCF. The Acceptance of Cosmetic Surgery Scale: Initial examination of its factor structure and correlates among Brazilian adults. Body Image. 2011;8:179-85.
19. Moreira GXS, Lorenzato L, Neufield CB, Almeida SSA. Brazilian Version of the Body Appreciation Scale (BAS) for Young Adolescents. Span J Psychol. 2018;21:e21.

20. Cooper PJ, Taylor MJ, Cooper Z, Fairburn CG. The Development and Validation of the Body Shape Questionnaire. Int J Eat Disord. 1987;6(4):485-94.

21. Conti MA, Cordás TA, Latorre MRDO. A study of the validity and reliability of the Brazilian version of the Body Shape Questionnaire (BSQ) among adolescents. Rev Bras Saúde Mater Infant. 2009;9(3):331-8.

22. Di Pietro M, Silveira DX. Internal validity, dimensionality and performance of the Body Shape Questionnaire in a group of Brazilian college students. Rev Bras Psiquiatr. 2009;31(1):21-4

23. Thompson JK, van den Berg P, Roehrig M, Guarda AS, Heinberg LJ. The Sociocultural Attitudes Towards Appearance Scale-3 (SATAQ-3): Development and Validation. Int J Eat Disord. 2004;35(3):293-304.

24. Amaral ACS, Conti MA, Ferreira MEC, Meireles JFF. Avaliação Psicométrica do Questionário de Atitudes Socioculturais em Relação à Aparência-3 (SATAQ-3) para Adolescentes. Psicol Teor Pesqui. 2015;31(4):471-9

25. Amaral ACS, Ribeiro MS, Conti MA, Ferreira CS, Ferreira MEC. Psychometric Evaluation of the Sociocultural Attitudes Towards Appearance Questionnaire-3 among Brazilian Young Adults. Span J Psychol. 2013;16:1-10.

26. Keery H, van den Berg P, Thompson JK. An evaluation of the Tripartite Influence Model of body dissatisfaction and eating disturbance with adolescent girls. Body Image. 20014;1(3):237-51.

27. Amaral ACS, Ferreira MEC, Scagliusi FB, Costa LS, Cordás TA, Conti MA. Avaliação psicométrica da Escala de Influência dos Três Fatores (EITF) de insatisfação corporal e transtorno alimentar para o idioma português. Psicol Reflex Crit. 2013;26(2):213-21.

28. Amaral ACS, Ferreira MEC. Body dissatisfaction and associated factors among Brazilian adolescents: A longitudinal study. Body Image. 2017;22:32-8.

29. Garner DM, Olmsted MP, Bohr Y, Garfinkel PE. The Eating Attitudes Test: psychometric features and clinical correlates. Psychol Med. 1982;12(4):871-7.

30. Bighetti F, Santos (B, Santos JE, Ribeiro RPP. Tradução e avaliação do Eating Attitudes Test em adolescentes do sexo feminino de Ribeirão Preto, São Paulo. J Bras Psiquiatr. 2004;53:339-46.

31. Rosenberg M. Society and the adolescent self-image. Middletown, CT: Wesleyan University Press; 1989.

32. Hutz CS, Zanon C. Revisão da adaptação, validação e normatização da Escala de Autoestima de Rosenberg. Aval Psicol. 2011:10:41-9.

33. Lobera IJ, Ríos PB. Spanish Version of the Body Appreciation Scale (BAS) for Adolescents. Span J Psychol. 2011;14(1):411-20.

34. Wood-Barcalow NL, Tylka TL, Augustus-Horvath CL. "But I like my body": Positive body image characteristics and a holistic model for young adult women. Body Image. 2010;7(2):106-16.

35. Skorek M, Song AV, Dunham Y. Self-Esteem as a Mediator between Personality Traits and Body Esteem: Path Analyses across Gender and Race/Ethnicity. PLos One. 2014;9(11):e112086.

36. Mendelson BK, Mendelson MJ, White DR. Body-Esteem Scale for adolescents and adults. J Personal Assess. 2001;76:90-106.

37. O'Dea JA. Body Image and Self-Esteem. In: Cash TF (Ed.). Encyclopedia of Body Image and Human Appearance. New York: Elsevier; 2012. p. 141-7.

38. Swami V, Weis L, Barron D, Furnham A. Positive body image is positively associated with hedonic (emotional) and eudaimonic (psychological and social) well-being in British adults. J Soc Psychol. 2018;158(5):541-52. 